\title{
Interacting phenotypes and the coevolutionary process: Interspecific indirect genetic effects alter coevolutionary dynamics
}

Running title: Trait interactions and coevolution

Stephen P. De Lisle ${ }^{1,2}$, Daniel I. Bolnick ${ }^{1}$, Edmund D. Brodie III $^{3}$, Allen J. Moore ${ }^{4}$, and Joel

W. McGlothlin ${ }^{5}$

Corresponding author: Stephen P. De Lisle Email: stephen.de_lisle@biol.lu.se

1. Department of Ecology \& Evolutionary Biology

University of Connecticut

75 N. Eagleville Road

Storrs, Connecticut, USA 06269

2 Present address:

Evolutionary Ecology Unit, Department of Biology

Lund University

Solvegatan 37, Lund, Sweden

3 Department of Biology and Mountain Lake Biological Station

University of Virginia

485 McCormick Road

Charlottesville, VA 22904 USA

4 Department of Entomology

University of Georgia

Athens, GA 30602 USA

5 Department of Biological Sciences

Virginia Tech

2125 Derring Hall, 926 West Campus Drive

Blacksburg, Virginia, USA 24060

Author Contributions All authors contributed to all aspects of the manuscript.

Acknowledgements Funding was provided by grants from the Royal Swedish Academy of

Sciences and Swedish Research Council to S. De Lisle (VR registration number 2019-

03706), the University of Connecticut and the NIAID (1R01AI123659-01A1) to D. Bolnick, and the National Science Foundation (DEB 1457463) to J. McGlothlin.

Data Accessibility No data to be archived 


\section{Interacting phenotypes and the coevolutionary process: Interspecific}

2 indirect genetic effects alter coevolutionary dynamics

3

4

5 Abstract Coevolution occurs when species interact to influence one another's fitness,

6 resulting in reciprocal evolutionary change. In many coevolving lineages, trait expression in

7 one species is modified by the genotypes and phenotypes of the other, forming feedback

8 loops reminiscent of models of intraspecific social evolution. Here, we adapt the theory of

9 within-species social evolution, characterized by indirect genetic effects and social selection

10 imposed by interacting individuals, to the case of interspecific interactions. In a trait-based

11 model, we derive general expressions for multivariate evolutionary change in two species and

12 the expected between-species covariance in evolutionary change when selection varies across

13 space. We show that reciprocal interspecific indirect genetic effects can dominate the

14 coevolutionary process and drive patterns of correlated evolution beyond what is expected

15 from direct selection alone. In extreme cases, interspecific indirect genetic effects can lead to coevolution when selection does not covary between species or even when one species lacks

17 genetic variance. Moreover, our model indicates that interspecific indirect genetic effects coevolution. Importantly, our model makes empirically testable predictions for how different forms of reciprocal interactions contribute to the coevolutionary process.

21

22 Key words: Coevolution, cross-species selection, interspecific indirect genetic effects,

23 quantitative genetics, species interactions 


\section{Introduction}

25 Coevolution occurs when interacting lineages evolve reciprocally in response to one another

26 (Janzen 1980, Thompson 1982). Although the concept of coevolution may be applied to

27 lineages that share genes, such as males and females of the same species (Arnqvist and Rowe

28 2002), it was originally invoked to explain patterns of correlated evolution between interacting species (Ehrlich and Raven 1964). In some cases, coevolution can result in tightly integrated mutualisms or spectacular arms races that drive the evolution of exceptional phenotypes (Brodie et al. 2002, Pellmyr 2003, Johnson and Anderson 2010). Yet even beyond these striking cases, coevolution is likely important for a wide range of interacting lineages, including consumers and their resources, hosts and their pathogens, competitors, and mutualists (Thompson 1982, 1994). Although coevolution has clearly played a major role in the origins of diversity, much is still unknown about when and how species interactions generate reciprocal evolutionary change. interactions between coevolving species and how selection imposed by one species manifests evolutionary change in an interacting species (Nuismer 2017). The interaction between species in coevolving lineages is often intimate, with one species spending a greater part of its life cycle in close contact with the other. Thus, coevolution bears a striking resemblance to intraspecific social evolution (Stearns 2012). Like social evolution, coevolution often includes interacting or extended phenotypes, which arise when traits can only be understood within the context of interactions with others (Dawkins 1982, Moore et al. 1997).

47 theory. First, the phenotype of one individual may cause fitness effects in a social partner,

48 leading to a form of selection known as social selection (West-Eberhard 1979, West- 
49 Eberhard 1983, West-Eberhard 1984, Wolf et al. 1999). At the heart of all coevolutionary models is a form of reciprocal fitness interaction that resembles social selection, where the

51 fitness of individuals in one species is influenced by traits in an interacting species (Brodie and Ridenhour 2003, Ridenhour 2005, Nuismer 2017). Second, models of social evolution may also include indirect genetic effects, which occur when the phenotype of one individual depends on the genotype of an interacting partner (Moore et al. 1997, Wolf et al. 1998). A classic example of indirect genetic effects is maternal effects, in which offspring phenotype is a function of both their own genes (a direct genetic effect) and maternal phenotypes such as litter size and provisioning (an indirect genetic effect) (Kirkpatrick and Lande 1989, Mousseau and Fox 1998, McAdam et al. 2002). When indirect genetic effects are reciprocal, feedback effects may inflate the genetic variance available for response to selection, drastically accelerating the rate of evolution (Moore et al. 1997). Although most models of indirect genetic effects do not extend beyond species boundaries, indirect genetic effects may also be common in species interactions between mutualists, competitors, parasites and hosts, and predators and prey. Phenotypic plasticity in response to an interacting species is common (Agrawal 2001), and when these influences on trait expression have a genetic basis they may represent interspecific indirect genetic effects (IIGEs; Shuster et al. 2006). Although IIGEs have received some attention in the context of community genetics (Shuster et al. 2006, Witham et al. 2020), their potential role in driving trait coevolution has been mostly unexplored.

To date, most explorations of IIGEs have been studies providing empirical support for their likely existence and their contribution to trait variation. Examples of interspecific phenotypic manipulation are common in nature (Table 1), and many of these cases can be argued to be putative cases of IIGEs. One possible example occurs in arbuscular mycorrhizae, where the genotypes of fungal mutualists can alter root traits in the plants they 
Table 1. Coevolutionary interactions where IIGEs may be prevalent.

\begin{tabular}{|c|c|c|c|}
\hline Interaction & IIGE synonym & $\begin{array}{l}\text { Diffuse/individual } \\
\text { interaction }\end{array}$ & $\begin{array}{l}\text { Example } \\
\text { References }\end{array}$ \\
\hline $\begin{array}{c}\text { host- } \\
\text { parasite }\end{array}$ & host manipulation & both & $\begin{array}{l}\text { (Thomas et } \\
\text { al. 2012) }\end{array}$ \\
\hline $\begin{array}{l}\text { predator- } \\
\text { prey }\end{array}$ & trait-mediated indirect interaction & both & $\begin{array}{c}\text { (Peacor and } \\
\text { Werner } \\
\text { 2001) }\end{array}$ \\
\hline $\begin{array}{l}\text { plant- } \\
\text { animal }\end{array}$ & $\begin{array}{l}\text { inducible direct defense } \\
\text { host-plant manipulations }\end{array}$ & both & $\begin{array}{c}\text { (Weis and } \\
\text { Abrahamson } \\
\text { 1986, Chen } \\
\text { 2008) }\end{array}$ \\
\hline $\begin{array}{l}\text { plant- } \\
\text { microbe }\end{array}$ & $\begin{array}{c}\text { joint trait } \\
\text { microbially mediated trait }\end{array}$ & diffuse & $\begin{array}{l}\text { (Friesen et } \\
\text { al. 2011, } \\
\text { O'Brien et } \\
\text { al. 2021) }\end{array}$ \\
\hline $\begin{array}{l}\text { host- } \\
\text { microbiome }\end{array}$ & $\begin{array}{l}\text { host control } \\
\text { host-microbiome interaction }\end{array}$ & diffuse & $\begin{array}{l}\text { (Stappenbeck } \\
\text { and Virgin } \\
\text { 2016, Foster } \\
\text { et al. 2017) }\end{array}$ \\
\hline $\begin{array}{l}\text { consumer- } \\
\text { resource }\end{array}$ & toxin sequestration & both & $\begin{array}{c}\text { (Züst et al. } \\
\text { 2018) }\end{array}$ \\
\hline
\end{tabular}

74 inhabit (Gianinazzi-Pearson et al. 2007). In host-parasite systems, parasite manipulation of

75 host traits (such as behavior) and reciprocal host manipulation of parasite traits (such as

76 growth rate, via immune response) are key features of species interactions (Thomas et al.

77 2012). Importantly, in many host-parasite systems, both the host and the parasite experience 78 sustained interactions with a small number of individuals of the other species, often over key

79 periods of the life history. For example, helminth parasites excrete a variety of

80 immunomodulatory products that suppress or misdirect the immune system of their

81 individual host (Damian 1997, Schmid-Hempel 2008, Oladiran and Belosevic 2012). Thus,

82 host immune response to infection is controlled by the genotype of both the host and parasite

83 (e.g., Barribeau 2014). As a specific example, some populations of threespine stickleback

84 (Gasterosteus aculeatus) initiate a strong immune response to infection by the cestode

85 Schistocephalus solidus (Fig. 1A) involving granulocyte proliferation and fibrosis, which 
86 effectively suppress cestode growth and viability (Weber et al. 2017). Other stickleback

87 populations do not exhibit this response and allow rapid cestode growth, perhaps representing

88 a tolerance strategy. Cestode growth is thus an indirect genetic effect of its host's genotype.

89 Conversely, the cestode has been shown to secrete compounds that suppress this host

90 response (Scharsack et al. 2004, 2007, 2013) and down-regulate sticklebacks' pro-fibrotic

91 gene expression (Fuess et al. 2020), suggesting reciprocal indirect genetic effects.

determined by genotypes of both fly and plant, and evolution of gall size is influenced in part

by cross-species selection imposed on Eurosta larvae by species at other trophic levels (Weis and Abrahamson 1986, Weis et al. 1992, Abrahamson and Weis 1997). This type of interaction is common across gall-forming insects and their plant hosts; in Hormaphis aphids, variation the bicycle gene has been linked to variation in gall size (Korgaonkar et al. 2021). In many herbivore-plant interactions, physical damage to leaves induces upregulation of defensive compounds to deter further herbivory, which can be countered by matching physiological changes in the herbivore (Ohgushi 2005). For example, Littorina snail herbivory changes foliar chemistry of the brown seaweed Ascophyllum nodosa (increased phlorotannin concentrations), which in turn reduces snail movement and consumption rates (Borell et al. 2004). intimate long-term associations between individuals. In contrast, predator-prey interactions tend to be more diffuse (Brodie and Brodie 1999). Prey sense predation risk through 


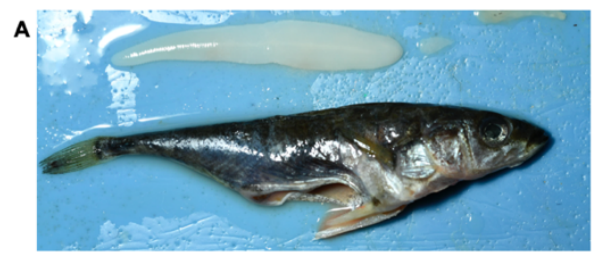

c

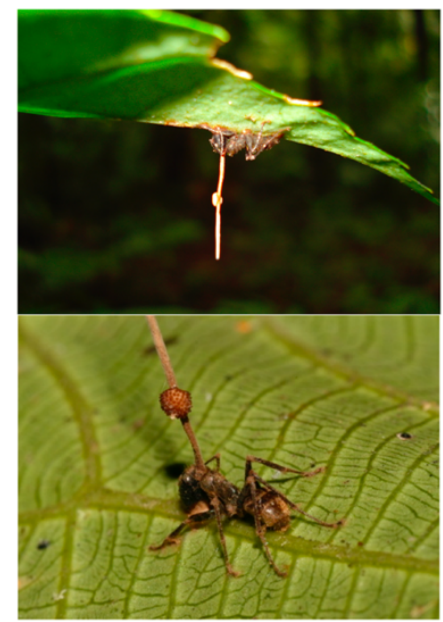

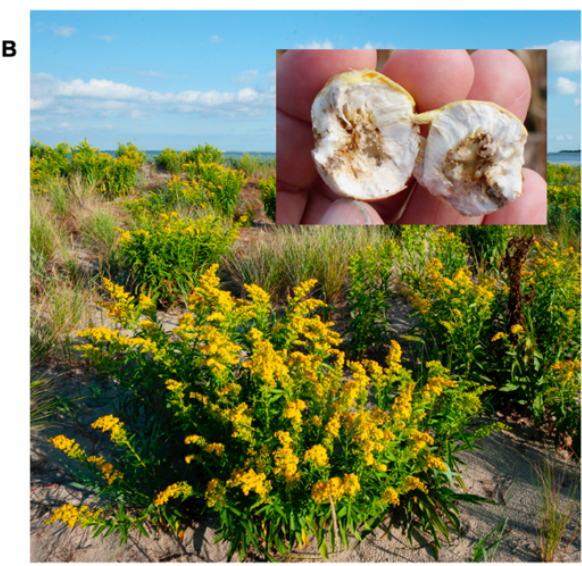

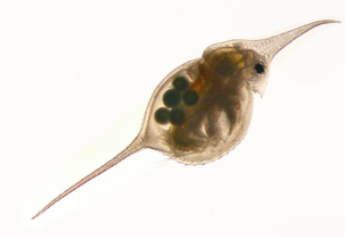

Figure 1. Examples of traits potentially mediated by IIGEs. IIGEs are a common feature of species interactions, particularly in host-parasite systems where prolonged contact between single individuals of both species determines fitness and trait expression for both. Here we highlight four examples where such IIGEs are likely contributing to trait expression and possibly patterns of among-population (co)variation. Panel A shows threespine stickleback fish (Gasterosteus aculeatus) and their cestode parasite Schistocephalus. Freshwater stickleback are the intermediate host for Schistocephalus, and each cestode acquires most of its lifetime resource pool while living inside a single host fish. Schistocephalus impose substantial reproductive and survival costs on hosts, and hosts have evolved an inducible (by the cestode) defense that suppresses cestode growth. Substantial among population-variation in this trait and in infection rates suggest these IIGEs may be mediating coevolution. Panel B shows goldenrod (Solidago) and a gall induced by the larvae (visible inside the gall) of the specialist gall-forming fly Eurosta. Gall size is induced by the genotype of the Eurosta larvae, and past work has shown that gall expression is a complex interaction between plant and fly genotype. Panel $\mathrm{C}$ shows the fungus Ophiocordyceps unilateralis, which manipulates behavior of its ant host prior to emergence of its fruiting body (Anderson et al. 2009). Panel D shows the water flea Daphnia lumholtzi, which induces growth of protective spines in response to chemical cues released by predatory fish (Agrawal 2001). Panel A and B main photos: S. De Lisle. Inset (B) photo: SriMesh / CC BY-SA (https://creativecommons.org/licenses/by-sa/3.0); C photo: "File:Ophiocordyceps unilateralis.png" by David P. Hughes, Maj-Britt Pontoppidan / CC BY 2.5; Panel D: "Water flea (Daphnia lumholtzi)" by Frupus / CC BY-NC 2.0. 
108

109

110

111

112

113

114

115

116

117

118

119

120

121

122

123

124

125

126

127

128

129

130

131

132

ways that mitigate their risk of predator encounter (Werner and Peacor 2003, Preisser et al. 2005). Perhaps the most prominent example is the tendency of some Daphnia genotypes to grow spines (Fig. 1D), or to migrate to other depths, when they detect scent cues (kairomones) from predatory fish (Weber and Declerck 1997, Boersma et al. 1998). These antipredator responses lead to systemic changes in gene expression and morphology (Tams et al. 2019), which are controlled in part by fish traits (e.g., production of a scent cue). These scents can themselves be variable and genetic, as illustrated by differences in how Daphnia respond to cues from landlocked versus anadromous alewife (Walsh and Post 2011). The Daphnia traits can thus be described resulting from IIGEs controlled by both Daphnia and fish genotypes. Unlike the intimate host-parasite interactions, however, prey may be responding to diffuse cues from a predator population as a whole.

Although IIGEs would appear to play a central role in trait interactions between many coevolving species, little is known about how these effects influence the dynamics of coevolution (Scheiner et al. 2015). Past approaches, which have included variancepartitioning models of community assembly (Shuster et al. 2006, Whitham et al. 2020) and models of "joint traits" expressed together by interacting species (Queller 2014, O’Brien et al. 2021), are suggestive of an important role for IIGEs in species interactions. However, we lack a general understanding of how reciprocal IIGEs may affect the coevolution of interacting phenotypes. The fact that indirect genetic effects within a species can create feedback loops and other complex evolutionary dynamics, including in the context of withinspecies coevolution (Drown and Wade 2014), suggests that IIGEs could play a major role in mediating trait coevolution between species. Coopting concepts from intraspecific social evolution theory, where trait-based IGEs are well developed, thus provides a natural way to understand the trait interactions that drive coevolution. Importantly, this trait-based approach allows the contribution of indirect genetic effects to the coevolutionary process to be fully 
explored. Our goal in this paper is to develop such models to provide a comprehensive theoretical assessment of how IIGEs contribute to coevolution between species. 1997, Wolf et al. 1999, McGlothlin et al. 2010) to the case of two interacting species. Our model applies to both pairwise interactions between individuals (e.g., stickleback and cestodes) as well as diffuse interactions between species mean values (e.g., predatory fish and selection, where phenotypes of individuals in one species influence fitness of individuals of another species (Figs. 1, 2). In addition to describing the contribution of these interspecific interactions to evolutionary change, we develop expressions for the among-population covariance in trait means across populations between two coevolving species. In the process, we formalize an interspecific analog of Zeng's (1988) quantitative genetic model of amongpopulation trait covariation, which we expand to incorporate interacting phenotypes. Our analysis shows that IIGEs may have a central role in driving and mediating coevolution.

\section{Reciprocal evolutionary change in interacting species}

151 To model coevolution in two interacting species, we first decompose trait expression into

152 three components: direct genetic effects, environmental effects, and indirect effects mediated by the phenotype of an interacting species. The phenotypic interface of coevolution involves traits with interacting effects across individuals of two species $x$ and $y$ and can be written as

$$
\begin{aligned}
& \mathbf{z}_{x}=\mathbf{a}_{x}+\mathbf{e}_{x}+\boldsymbol{\Phi}_{x y} \mathbf{z}_{y}^{\prime} \\
& \mathbf{z}_{y}=\mathbf{a}_{y}+\mathbf{e}_{y}+\boldsymbol{\Phi}_{y x} \mathbf{z}_{x}^{\prime},
\end{aligned}
$$


157 where $\mathbf{z}_{i}$ is a column vector of $m$ traits expressed in an individual of species $i=x$ or $y$ ( $m$ is

158 not necessarily equal in each species), $\mathbf{a}_{i}$ is the corresponding column vector of direct genetic

159 effects, and $\mathbf{e}_{i}$ is an uncorrelated vector of residual environmental effects; primes denote traits

160 of interacting individuals of another species. The matrix $\boldsymbol{\Phi}_{x y}$ quantifies the effect of traits in

161 an interacting individual of species $y\left(\mathbf{z}_{y}^{\prime}\right)$ on the expression of traits in a focal individual of

162 species $x$ (Fig. 2, Table 2), while the matrix $\boldsymbol{\Phi}_{y x}$ quantitifies such effects in the opposite

163 direction. Thus, $\boldsymbol{\Phi}_{i j}$ is an interspecific analog of the matrix of conspecific indirect genetic

164 effects, $\boldsymbol{\Psi}$ (Moore et al. 1997). The individual elements of $\boldsymbol{\Phi}_{i j}$, which we write as $\phi_{i j}^{k l}$,

165 represent partial regression coefficients of trait $l$ in species $j$ on trait $k$ in species $i$. When

166 traits are standardized to the same scale, these coefficients will typically be limited to a range

167 of -1 to 1 . Although we focus on interactions between pairs of individuals for simplicity of

168 notation, this model could be easily extended to describe interactions with multiple

169 individuals (cf. McGlothlin et al. 2010). For more diffuse interactions (e.g., alewife and

170 Daphnia), the elements of $\boldsymbol{\Phi}_{i j}$ could represent a weighted average of effects from integrating

171 across the phenotype distribution of the interacting population. 


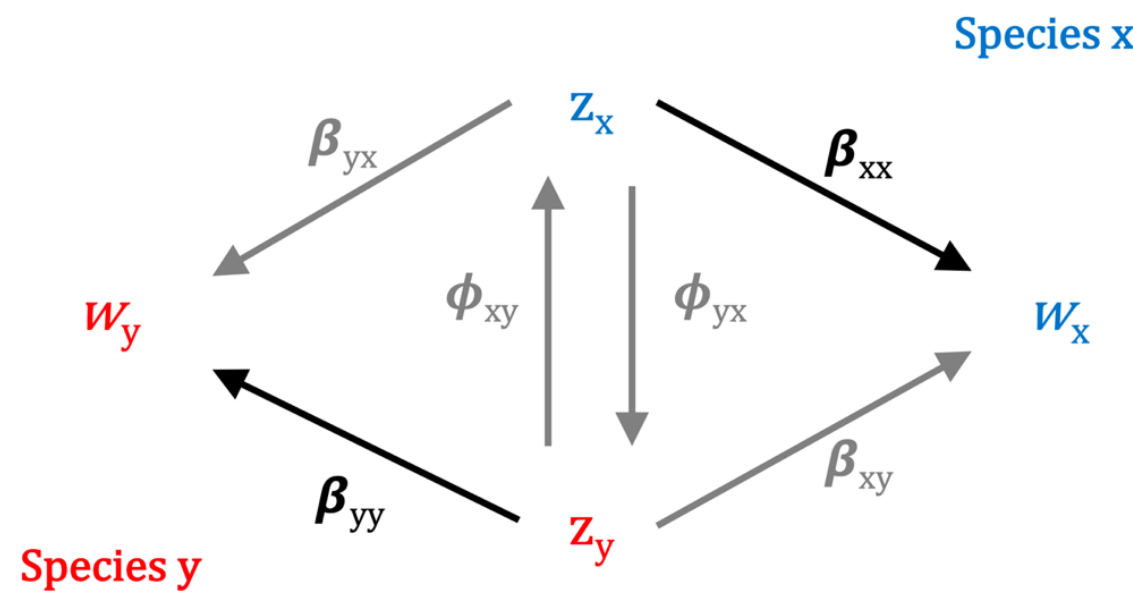

Figure 2. Path model of selection when traits and fitness are determined by interactions with heterospecifics. Individual trait values of two interacting species, $x$ (blue) and $y$ (red), are represented by $z$. Traits directly influence individual relative fitness $(w)$ of the species that express them via natural selection ( $\beta_{x x}$ and $\beta_{y y}$, black arrows). Traits can also reciprocally influence expression of traits of heterospecific social partners, via interspecific indirect genetic effects $\Phi$. Traits can also influence fitness of heterospecific social partners, via cross-species selection ( $\beta_{x y}$ and $\beta_{y x}$, grey arrows). Social effects are illustrated in grey arrows, direct effects in black. 
Table 2. Definition of key parameters and expressions.

\begin{tabular}{c|l}
\hline Expression / parameter & \multicolumn{1}{c}{ Biological definition } \\
\hline $\mathbf{z}_{x}, \mathbf{z}_{y}$ & $\begin{array}{l}\text { Vectors of individual trait values for species } x \\
\text { and } y, \text { with a prime when present denoting } \\
\text { partner traits. }\end{array}$ \\
\hline $\mathbf{a}_{x}, \mathbf{a}_{y}$ & $\begin{array}{l}\text { Vectors of individual breeding values for } \\
\text { species } x \text { and } y \text {. }\end{array}$ \\
\hline $\boldsymbol{\Phi}_{x y}, \boldsymbol{\Phi}_{y x}$ & $\begin{array}{l}\text { Interspecific indirect effects (in matrix form) } \\
\text { The effect of traits in individuals of species } y \text { on } \\
\text { trait expression in species } x \text {, and vice-versa. }\end{array}$ \\
\hline $\boldsymbol{\beta}_{x x}, \boldsymbol{\beta}_{y y}$ & $\begin{array}{l}\text { Vectors of within-species natural selection } \\
\text { gradients for each species. }\end{array}$ \\
\hline $\boldsymbol{\beta}_{x y}, \boldsymbol{\beta}_{y x}$ & $\begin{array}{l}\text { Vectors of cross-species selection gradients for } \\
\text { each species. The effects of traits in species } y \\
\text { on fitness of individual species } x, \text { and vice- } \\
\text { versa. }\end{array}$ \\
\hline $\mathbf{G}_{x x}, \mathbf{G}_{y y}$ & $\begin{array}{l}\text { Genetic covariance matrices of traits for species } \\
x \text { and } y \text {. }\end{array}$ \\
\hline $\mathbf{G}_{x y}, \mathbf{G}_{y x}$ & $\begin{array}{l}\text { Covariance matrices for breeding values of } \\
\text { interacting individuals of species } x \text { and } y \text { within } \\
\text { a population. }\end{array}$ \\
\hline $\left.\operatorname{Cov}\left(\beta_{x x}, \beta_{y y}\right), \Delta \bar{z}_{y}\right)$ & $\begin{array}{l}\text { Coevolutionary covariance, defined as the } \\
\text { covariance in evolutionary change between } \\
\text { interacting species, across populations. When } \\
\text { standardized by the variances in evolutionary } \\
\text { response for each species, becomes a scale-free } \\
\text { correlation. } \\
\text { Between-species, among-population covariance } \\
\text { in selection gradients (shown here for within- } \\
\text { species selection) }\end{array}$ \\
\hline & \\
\hline &
\end{tabular}



phenotype vector for each species is

174

$$
\begin{gathered}
\overline{\mathbf{z}}_{x}=\overline{\mathbf{a}}_{x}+\boldsymbol{\Phi}_{x y} \overline{\mathbf{z}}_{y} \\
\overline{\mathbf{z}}_{y}=\overline{\mathbf{a}}_{y}+\boldsymbol{\Phi}_{y x} \overline{\mathbf{z}}_{x} .
\end{gathered}
$$

176 Noting the change in the mean additive genetic value in each species is $\Delta \overline{\mathbf{a}}=\operatorname{Cov}(\mathbf{a}, w)$

177 (Robertson 1966, Price 1970, 1972) where $w$ is relative fitness, we can now define

178 evolutionary change in the multivariate mean phenotype as

179

$$
\begin{aligned}
\Delta \overline{\mathbf{z}}_{x} & =\operatorname{Cov}\left(\mathbf{a}_{x}, w_{x}\right)+\boldsymbol{\Phi}_{x y} \Delta \overline{\mathbf{z}}_{y} \\
\Delta \overline{\mathbf{z}}_{y} & =\operatorname{Cov}\left(\mathbf{a}_{y}, w_{y}\right)+\boldsymbol{\Phi}_{y x} \Delta \overline{\mathbf{z}}_{x} .
\end{aligned}
$$

In equation (3), the first term on each right-hand side describes direct change due to natural selection on the focal species, and the second term describes indirect change due to the product of IIGEs and phenotypic change in the interacting species. This second term is analogous to transmission bias (Fisher and McAdam 2019), and in this case, specifically describes changes in one species that are induced by evolution in an interacting species. It can be seen from equation (3) that species will coevolve whenever there are IIGEs in both species, because the change in phenotypic mean in species $x$ depends upon the change of mean in species $y$ and vice versa whenever both $\boldsymbol{\Phi}_{i j} \neq \mathbf{0}$. Expanding equation (3) by substitution results in explicit equations for evolutionary change in each species,

$$
\Delta \overline{\mathbf{z}}_{x}=\left(\mathbf{I}_{x}-\boldsymbol{\Phi}_{x y} \boldsymbol{\Phi}_{y x}\right)^{-1}\left[\operatorname{Cov}\left(\mathbf{a}_{x}, w_{x}\right)+\boldsymbol{\Phi}_{x y} \operatorname{Cov}\left(\mathbf{a}_{y}, w_{y}\right)\right]
$$




$$
\Delta \overline{\mathbf{z}}_{y}=\left(\mathbf{I}_{y}-\boldsymbol{\Phi}_{y x} \boldsymbol{\Phi}_{x y}\right)^{-1}\left[\operatorname{Cov}\left(\mathbf{a}_{y}, w_{y}\right)+\boldsymbol{\Phi}_{y x} \operatorname{Cov}\left(\mathbf{a}_{x}, w_{x}\right)\right] .
$$

191

192

where $\mathbf{I}_{x}$ and $\mathbf{I}_{y}$ are identity matrices with dimensionality equal to the number of traits in the

193

two species and the multiplier $\left(\mathbf{I}-\boldsymbol{\Phi}_{i j} \boldsymbol{\Phi}_{j i}\right)^{-1}$ quantifies the feedback effect of reciprocal

IIGEs. Equation 4 illustrates that total evolutionary change in a species is determined both

by change in the breeding value of that species $\left(\operatorname{Cov}\left(\mathbf{a}_{i}, w_{i}\right)\right)$ and by change in the breeding

value of the interacting species, mediated by IIGEs $\left(\boldsymbol{\Phi}_{i j} \operatorname{Cov}\left(\mathbf{a}_{j}, w_{j}\right)\right)$. Whenever IIGEs occur

in both species such that $\boldsymbol{\Phi}_{i j} \boldsymbol{\Phi}_{j i} \neq \mathbf{0}$, this multiplier alters the total amount of evolutionary

change in both species. In order for such an effect to arise, there must be a feedback loop in

phenotypic expression. The simplest of these arises when there are two traits with reciprocal

IIGEs, such that trait $k$ in species $i$ affects trait $l$ in species $j$ and trait $l$ in turn influences the

201

expression of trait $k$. In general, when $\phi_{i j}^{k l}$ and $\phi_{j i}^{l k}$ are of the same sign, the magnitude of

evolutionary change will be enhanced, and when they are of opposite signs, evolutionary

change will be diminished (Fig. 3).

$$
\begin{gathered}
w_{x}=\alpha_{x}+\mathbf{z}_{x}^{\mathrm{T}} \boldsymbol{\beta}_{x x}+\mathbf{z}_{y}^{\prime \mathrm{T}} \boldsymbol{\beta}_{x y}+\varepsilon_{x} \\
w_{y}=\alpha_{y}+\mathbf{z}_{y}^{\mathrm{T}} \boldsymbol{\beta}_{y y}+\mathbf{z}_{x}^{\prime \mathrm{T}} \boldsymbol{\beta}_{y x}+\varepsilon_{y}
\end{gathered}
$$

where $w$ is individual relative fitness, $\alpha$ is an intercept, $\varepsilon$ is an error term, and the superscript

209 T denotes transposition. Directional selection in each species is partitioned into two selection gradients. First, the within-species selection gradients $\left(\boldsymbol{\beta}_{x x}\right.$ and $\left.\boldsymbol{\beta}_{y y}\right)$ describe the direct

211 effects of an individual's traits on its own fitness (Fig. 2; Lande and Arnold 1983). The 
cross-species selection gradients $\left(\boldsymbol{\beta}_{x y}\right.$ and $\left.\boldsymbol{\beta}_{y x}\right)$ relate the fitness of a focal individual of one

213 species ( $x$ or $y)$ to the traits of individuals of the other coevolving species ( $y$ or $x$ ) (Fig. 2).

214 Both of these are linear components of selection; we consider nonlinear terms (interactions

215 for fitness between focal and partner traits) below (see Incorporating Specific Fitness

216 Models). The cross-species selection gradient is analogous to the directional social selection

217 gradient in within-species models (Wolf et al. 1999) and if pairs or groups of interacting

218 individuals can be identified in a natural population, can be estimated in a similar way (cf.

219 Ridenhour 2005).

$$
\begin{gathered}
\Delta \overline{\mathbf{z}}_{x}=\left(\mathbf{I}_{x}-\boldsymbol{\Phi}_{x y} \boldsymbol{\Phi}_{y x}\right)^{-1}\left[\operatorname{Cov}\left(\mathbf{a}_{x}, \mathbf{z}_{x}^{\mathrm{T}}\right) \boldsymbol{\beta}_{x x}+\operatorname{Cov}\left(\mathbf{a}_{x}, \mathbf{z}_{y}^{\prime \mathrm{T}}\right) \boldsymbol{\beta}_{x y}+\right. \\
\left.\boldsymbol{\Phi}_{x y} \operatorname{Cov}\left(\mathbf{a}_{y}, \mathbf{z}_{y}^{\mathrm{T}}\right) \boldsymbol{\beta}_{y y}+\boldsymbol{\Phi}_{x y} \operatorname{Cov}\left(\mathbf{a}_{y}, \mathbf{z}_{x}^{\prime \mathrm{T}}\right) \boldsymbol{\beta}_{y x}\right]
\end{gathered}
$$

$$
\begin{gathered}
\Delta \overline{\mathbf{z}}_{y}=\left(\mathbf{I}_{y}-\boldsymbol{\Phi}_{y x} \boldsymbol{\Phi}_{x y}\right)^{-1}\left[\operatorname{Cov}\left(\mathbf{a}_{y}, \mathbf{z}_{y}^{\mathrm{T}}\right) \boldsymbol{\beta}_{y y}+\operatorname{Cov}\left(\mathbf{a}_{x}, \mathbf{z}_{x}^{\mathrm{T}}\right) \boldsymbol{\beta}_{y x}+\right. \\
\left.\boldsymbol{\Phi}_{y x} \operatorname{Cov}\left(\mathbf{a}_{x}, \mathbf{z}_{x}^{\mathrm{T}}\right) \boldsymbol{\beta}_{x x}+\boldsymbol{\Phi}_{y x} \operatorname{Cov}\left(\mathbf{a}_{x}, \mathbf{z}_{y}^{\prime \mathrm{T}}\right) \boldsymbol{\beta}_{x y}\right]
\end{gathered}
$$

225 Each of these equations consists of four terms representing four components of the total

226 response to selection. The first term $\left(\operatorname{Cov}\left(\mathbf{a}_{x}, \mathbf{z}_{x}^{\mathrm{T}}\right) \boldsymbol{\beta}_{x x}\right)$ represents response to within-species selection $\left(\boldsymbol{\beta}_{x x}\right.$ or $\left.\boldsymbol{\beta}_{y y}\right)$, the second $\left(\operatorname{Cov}\left(\mathbf{a}_{x}, \mathbf{z}_{y}^{\prime T}\right) \boldsymbol{\beta}_{x y}\right)$ represents response to cross-species

228 linear selection $\left(\boldsymbol{\beta}_{x y}\right.$ or $\left.\boldsymbol{\beta}_{y x}\right)$, and the last two terms $\left(\boldsymbol{\Phi}_{x y} \operatorname{Cov}\left(\mathbf{a}_{y}, \mathbf{z}_{y}^{\mathrm{T}}\right) \boldsymbol{\beta}_{y y}+\right.$ the interacting species. Note that the third and fourth terms of the first part of equation (6a) 
shows that the change in response to within-species selection depends on the covariance of phenotype of the opposite species.

$$
\Delta \overline{\mathbf{z}}_{x}=\left(\mathbf{I}_{x}-\boldsymbol{\Phi}_{x y} \boldsymbol{\Phi}_{y x}\right)^{-1}\left[\left(\mathbf{G}_{x x}+\mathbf{G}_{x y} \boldsymbol{\Phi}_{x y}^{\mathrm{T}}\right)\left(\mathbf{I}_{x}-\boldsymbol{\Phi}_{y x}^{\mathrm{T}} \boldsymbol{\Phi}_{x y}^{\mathrm{T}}\right)^{-1} \boldsymbol{\beta}_{x x}+\right.
$$

$$
\left(\mathbf{G}_{x y}+\mathbf{G}_{x x} \boldsymbol{\Phi}_{y x}^{\mathrm{T}}\right)\left(\mathbf{I}_{y}-\boldsymbol{\Phi}_{x y}^{\mathrm{T}} \boldsymbol{\Phi}_{y x}^{\mathrm{T}}\right)^{-1} \boldsymbol{\beta}_{x y}+
$$

$$
\begin{gathered}
\boldsymbol{\Phi}_{x y}\left(\mathbf{G}_{y y}+\mathbf{G}_{y x} \boldsymbol{\Phi}_{y x}^{\mathrm{T}}\right)\left(\mathbf{I}_{y}-\boldsymbol{\Phi}_{x y}^{\mathrm{T}} \boldsymbol{\Phi}_{y x}^{\mathrm{T}}\right)^{-1} \boldsymbol{\beta}_{y y}+ \\
\left.\boldsymbol{\Phi}_{x y}\left(\mathbf{G}_{y x}+\mathbf{G}_{y y} \boldsymbol{\Phi}_{x y}^{\mathrm{T}}\right)\left(\mathbf{I}_{x}-\boldsymbol{\Phi}_{y x}^{\mathrm{T}} \boldsymbol{\Phi}_{x y}^{\mathrm{T}}\right)^{-1} \boldsymbol{\beta}_{y x}\right]
\end{gathered}
$$

where $\mathbf{G}_{x x}$ and $\mathbf{G}_{y y}$ represent within-species genetic (co)variance matrices and $\mathbf{G}_{x y}$ and $\mathbf{G}_{y x}$ represent cross-species genetic covariance. Note that in a model of pairwise interactions, $\mathbf{G}_{y x}=\mathbf{G}_{x y}^{\mathrm{T}}$. between the species within the population of interest. This covariance may arise via any mechanism that leads to nonrandom genetic assortment between species $x$ and $y$ with respect 
such assortment including behavioral preference for certain traits in heterospecific partners,

257 fine-scale population structure, habitat preference, and vertical transmission of symbionts.

258 We elaborate on the contribution of $\mathbf{G}_{x y}$ and $\boldsymbol{\Phi}$ to phenotypic assortment between individuals of interacting species $\left(C_{x y}\right)$ in equation (A1). However, there is currently limited evidence of this type of interspecific genetic assortment, as few investigators seemed to have attempted to measure such a covariance. We therefore investigate dynamics in both the presence and absence of such assortment in our model development below. variance, $\mathbf{G}_{y y}$ or $\mathbf{G}_{x x}$, and $\mathbf{G}_{x y} \boldsymbol{\Phi}_{x y}^{\mathrm{T}}$ or $\mathbf{G}_{y x} \boldsymbol{\Phi}_{y x}^{\mathrm{T}}$, which represent an interaction between IIGEs and cross-species genetic covariance. Response to cross-species selection depends on the sum of cross-species genetic variance and $\mathbf{G}_{x x} \boldsymbol{\Phi}_{y x}^{\mathrm{T}}$ or $\mathbf{G}_{y y} \boldsymbol{\Phi}_{x y}^{\mathrm{T}}$, which describes the genetic variance created by IIGEs. Each term in equation (7) also contains an additional feedback

268 multiplier, $\left(\mathbf{I}_{y}-\boldsymbol{\Phi}_{x y}^{\mathrm{T}} \boldsymbol{\Phi}_{y x}^{\mathrm{T}}\right)^{-1}$, which further enhances the response to selection when there

269 are reciprocal IIGEs. Because of this additional multiplier, when IIGEs are reciprocal and of

270 the same sign, their effects on can be massive, mirroring the effects observed for within-

271 species IGEs (Moore et al. 1997, McGlothlin et al. 2010). As in previous equations, the last

272 two terms in equation (7) represent a sort of evolutionary feedback that occurs across

273 generations and is only present when there are IIGEs. These effects of $\boldsymbol{\Phi}$ and cross-species

274 selection on evolutionary response are illustrated in Fig. 3. 



Figure 3. Reciprocal IIGEs and cross-species selection change evolutionary response in a single species. Panels show the separate effects on evolutionary response in species $\mathrm{x}$ of indirect genetic effects (Panel A) and cross-species selection with genetic assortment (Panel B). Panel A shows the effects of reciprocal IIGEs holding all other evolutionary parameters constant, and assuming no cross-species selection. Panel B shows the effects of cross-species selection imposed by species y on species $\mathrm{x}$, in combination with the genetic assortment between interactants of the different species, and assuming IIGEs are absent. In both panels, $G_{x}=G_{y}=1, \beta_{y y}=\beta_{x x}=1$.

\section{Correlated evolution between interacting species: the coevolutionary covariance}

A key feature of coevolving lineages is correlated evolution across populations subject to varying ecological conditions. Here, we seek to understand how the equations of selection response can be used to understand how selection and IIGEs contribute to this shared amongpopulation divergence. We can explore the contribution of interspecific social effects to correlated evolution between interacting species by solving for the covariance in evolutionary change between interacting species, $\operatorname{Cov}\left(\Delta \bar{z}_{x}, \Delta \bar{z}_{y}\right)$, which we call the coevolutionary covariance (with the caveat that this covariance also reflects changes due in part to effects of IIGEs). Such a covariance represents the expected pattern of reciprocal phenotypic change through time in a single pair of populations of two species experiencing fluctuating selection, or perhaps more importantly, across a set of populations in space under varying selection relevance to understanding geographic variation among populations. 
The coevolutionary covariance reflects the degree to which divergence among

population means in two species has occurred jointly. High absolute values of the

coevolutionary covariance indicate tightly-coupled coevolutionary change between the two

species, whereas values around zero indicate that evolutionary change occurs independently.

292 When scaled by the total amount of population divergence (variances in $\Delta \bar{z}_{x}$ and $\Delta \bar{z}_{y}$ ), this

293 becomes a scale-free correlation describing the proportion of total divergence shared between

294 the two species. Importantly, we expect the coevolutionary covariance to be often be related to the covariance in population means, $\operatorname{Cov}\left(\bar{z}_{x}, \bar{z}_{y}\right) \propto \operatorname{Cov}\left(\Delta \bar{z}_{x}, \Delta \bar{z}_{y}\right)$ because, for example,

$\operatorname{Cov}\left(\bar{z}_{x}, \bar{z}_{y}\right)^{t+1}=\operatorname{Cov}\left(\bar{z}_{x}, \bar{z}_{y}\right)^{t}+\operatorname{Cov}\left(\Delta \bar{z}_{x}, \Delta \bar{z}_{y}\right)$ (under the assumption $\operatorname{Cov}\left(\Delta \bar{z}_{x}, \bar{z}_{y}\right)+$

$\left.\operatorname{Cov}\left(\bar{z}_{x}, \Delta \bar{z}_{y}\right)=0\right)$. Thus, expanding $\operatorname{Cov}\left(\Delta \bar{z}_{x}, \Delta \bar{z}_{y}\right)$ allows the possibility to assess how phenotypes.

To simplify our analysis, we use models of a single trait in each species. Developing

a full equation including all sources of covariance quickly becomes cumbersome, so we focus on three instructive special cases that illustrate the explicit impacts of considering crossspecies selection and interspecific interacting phenotypes. The simplest case occurs when

$$
\operatorname{Cov}\left(\Delta \bar{z}_{x}, \Delta \bar{z}_{y}\right)=G_{x x} G_{y y} \operatorname{Cov}\left(\beta_{x x}, \beta_{y y}\right)
$$

310 Equation (8) represents what is usually thought of as one source of correlated evolution

311 between interacting species: predictable variation in the type of selection occurring across 
space. Empirical data suggest that selection varies substantially in magnitude across space

313 (Siepielski et al. 2013) and that spatially autocorrelated biotic selection plays a substantial

314 role in driving divergence in trait means (Urban et al. 2011). Importantly, not just any

315 variance in selection will do. In order to create covariance in evolutionary change, selection

316 must covary between the interacting species. Cross-species selection, however, does not play

317 a role in equation (8) because it does not contribute to an evolutionary response to selection

318 in the absence of IIGEs and cross-species genetic covariance. Equation (8) is analogous to

319 the results of Zeng's (1988) model of correlated trait evolution under directional selection,

320 although for the case of traits expressed in different species. leads to three new sources of covariance among populations:

$$
\begin{gathered}
\operatorname{Cov}\left(\Delta \bar{z}_{x}, \Delta \bar{z}_{y}\right)=G_{x x} G_{y y} \operatorname{Cov}\left(\beta_{x x}, \beta_{y y}\right)+G_{x y}^{2} \operatorname{Cov}\left(\beta_{y x}, \beta_{x y}\right)+ \\
G_{x x} G_{x y} \operatorname{Cov}\left(\beta_{x x}, \beta_{y x}\right)+G_{y y} G_{x y} \operatorname{Cov}\left(\beta_{y y}, \beta_{x y}\right) .
\end{gathered}
$$

326 The second term in equation (9) $\left(G_{x y}^{2} \operatorname{Cov}\left(\beta_{y x}, \beta_{x y}\right)\right)$ shows that cross-species selection will contribute to the coevolutionary covariance only when there is cross-species genetic covariance for the traits that affect fitness. Such covariances occur if, for example, parasite and host genotypes associate nonrandomly for the traits that influence their partner's fitness.

330 The third and fourth terms $\left(G_{x x} G_{x y} \operatorname{Cov}\left(\beta_{x x}, \beta_{y x}\right)+\left(G_{y y} G_{x y} \operatorname{Cov}\left(\beta_{y y}, \beta_{x y}\right)\right)\right.$ represent a

331 relationship between the effect of species on itself and on its heterospecific partner. These

332 will be nonzero if populations with strong within-species selection also exhibit strong cross333 species selection. 


$$
\begin{gathered}
\operatorname{Cov}\left(\Delta \bar{z}_{x}, \Delta \bar{z}_{y}\right)=U\left[G_{x x} G_{y y} \operatorname{Cov}\left(\beta_{x x}, \beta_{y y}\right)+\right. \\
G_{x x}^{2} \phi_{y x} \operatorname{Var}\left(\beta_{x x}\right)+G_{y y}^{2} \phi_{x y} \operatorname{Var}\left(\beta_{y y}\right)+ \\
G_{x x} G_{y y} \phi_{x y} \phi_{y x} \operatorname{Cov}\left(\beta_{x x}, \beta_{y y}\right)+G_{x x} G_{y y} \phi_{x y} \phi_{y x} \operatorname{Cov}\left(\beta_{x y}, \beta_{y x}\right)+ \\
G_{x x} G_{y y} \phi_{x y} \operatorname{Cov}\left(\beta_{x x}, \beta_{y x}\right)+G_{x x} G_{y y} \phi_{y x} \operatorname{Cov}\left(\beta_{y y}, \beta_{x y}\right)+ \\
\left.2 G_{x x}^{2} \phi_{y x}^{2} \operatorname{Cov}\left(\beta_{x x}, \beta_{x y}\right)+2 G_{y y}^{2} \phi_{x y}^{2} \operatorname{Cov}\left(\beta_{y y}, \beta_{y x}\right)+\mathcal{V}\right],
\end{gathered}
$$

where feedback effects of IIGEs are described by $\mathcal{U}=\left(1-\phi_{x y} \phi_{y x}\right)^{-4}$ and $\mathcal{V}$ collects negligible third- and fourth-order $\phi$ terms:

$$
\begin{gathered}
\mathcal{V}=G_{x x}^{2} \phi_{y x}^{3} \operatorname{Var}\left(\beta_{x y}\right)+G_{y y}^{2} \phi_{x y}^{3} \operatorname{Var}\left(\beta_{y x}\right)+ \\
G_{x x} G_{y y} \phi_{x y} \phi_{y x}^{2} \operatorname{Cov}\left(\beta_{y y}, \beta_{x y}\right)+G_{x x} G_{y y} \phi_{x y}^{2} \phi_{y x} \operatorname{Cov}\left(\beta_{y x}, \beta_{x x}\right)+ \\
G_{x x} G_{y y} \phi_{x y}^{2} \phi_{y x}^{2} \operatorname{Cov}\left(\beta_{y x}, \beta_{x y}\right) .
\end{gathered}
$$

344 The first term in equation (10) is identical to equation (8) and represents covariance in direct 345 within-species selection. The second $\left(G_{x x}^{2} \phi_{y x} \operatorname{Var}\left(\beta_{x x}\right)\right)$ and third $\left(G_{y y}^{2} \phi_{x y} \operatorname{Var}\left(\beta_{y y}\right)\right)$ terms 



Figure 4. IIGEs drive and mediate coevolution between interacting species. Panels show the coevolutionary correlation between two interacting species as a function of the indirect genetic effect parameters $\phi$, from equation (10) standardized by the evolutionary rates. In the absence of social interaction effects, correlated evolution (coevolution) between species is driven entirely by covariance in natural selection between the species (see text). Reciprocal IIGEs can generate coevolution even when there is not covariance in natural selection (Panel A), and can even drive coevolution even when one species lacks genetic variance (Panel B). IIGEs modify observed coevolutionary patterns when natural selection does covary $\left(\operatorname{Cov}\left(\beta_{x x}, \beta_{y y}\right)=0.5\right.$; Panel $\left.C\right)$. When cross-species selection and IIGEs act together, coevolutionary patterns are a complex third order polynomial (Panel D; $\operatorname{Cov}\left(\beta_{x y}, \beta_{y x}\right)=0.5$, $\left.\operatorname{Var}\left(\beta_{x y}\right)=\operatorname{Var}\left(\beta_{y x}\right)=1\right)$. When natural and cross-species selection both act $\left(\operatorname{Var}\left(\beta_{x y}\right)=\right.$ $\left.\operatorname{Var}\left(\beta_{\mathrm{yx}}\right)=\operatorname{Var}\left(\beta_{y y}\right)=\operatorname{Var}\left(\beta_{x x}\right)=1\right)$ and covary positively $(0.5)$, (Panel E), effects of IIGEs become stronger in comparison to the case of (co)variance in natural selection alone. When covariance between natural and cross-species selection is negative $(-0.8)$ a ridge is observed (Panel F). 
347 show that in the presence of IIGEs, simple variance in within-species selection across

348 populations can generate a coevolutionary covariance (or correlation, Fig. 4A; corresponding

349 evolutionary rates and covariances are plotted in Figs. S1 and S2, respectively). This

350 covariance in evolutionary response occurs as a necessary consequence of the dependence of

351 the trait mean of one species on that of the other. Thus, in the presence of IIGEs, a

coevolutionary covariance may occur even when selection is uncorrelated between the species has genetic variation (Fig. 4B). species exist in a feedback loop, the effect of covariance in within-species selection is amplified if $\phi_{x y}$ and $\phi_{y x}$ are of the same sign and diminished if they are of opposite signs.

359 The combined effects of the second, third, and fourth terms lead to a complex relationship between IIGEs and the coevolutionary covariance when within-species selection varies (Fig. 4C). In some cases, IIGEs can even reverse the sign of the correlation that would be expected in their absence (Fig. 4C). In addition, reciprocal IIGEs may cause covariance in cross-species selection to contribute to the coevolutionary covariance, causing an even more complex relationship (Fig. 4D). the two species through their effects on the coevolutionary covariance, this can occur both when there is covariance in gradients across species (terms 6 and 7) and when there is covariance in gradients in the same species (terms 8 and 9). The total effect of IIGEs may be 
371 quite complex when cross-species selection varies, with subtle changes in $\phi$ resulting in

372 dramatic changes in the expected among population correlation (Fig. 4D, E-F).

An equation incorporating both IIGEs and $G_{x y}$ quickly becomes unwieldy, but we present a compact form containing 10 (co)variance terms as equation (A2), which illustrates influence coevolution.

\section{Incorporating specific fitness models}

379 Coevolutionary models often posit complex relationships between interacting phenotypes and fitness (Nuismer 2017). Although the selection model we present here is linear, more complex relationships can be incorporated by translating specific fitness functions to selection gradients. The adaptive landscape represents the theoretical relationship between a population's mean fitness and its phenotypic mean (Arnold et al. 2001). Selection gradients represent the partial slope of the adaptive landscape with respect to a given phenotype, and thus the multivariate selection gradient may be calculated using a vector of partial derivatives

386 if the adaptive landscape can be written as a differentiable function (Lande 1979, Lande and

387 Arnold 1983). In many cases, the multivariate selection gradient may be calculated using

388 partial derivatives of the individual fitness function as well (Lande and Arnold 1983, Abrams et al. 1993, McGlothlin et al. 2021), evaluated over the phenotypic distribution (Phillips and Arnold 1989). Once selection gradients have been calculated for a given model, they may be

391 substituted into equations (6-7) to explore the effects of a given fitness function on selection

392 response and the coevolutionary covariance (see also Brodie and Ridenhour 2003). This 
393

394

395

396

397

398

399

400

401

402

403

404

405

$$
\begin{gathered}
\operatorname{Cov}\left(\Delta \bar{z}_{x}, \Delta \bar{z}_{y}\right)=\mathcal{U}\left[G_{x x} G_{y y}\left(1-\phi_{x y}-\phi_{y x}+2 \phi_{x y} \phi_{y x}\right) \operatorname{Cov}\left(b_{x}, b_{y}\right)+\right. \\
\left.G_{x x}^{2} \phi_{y x}\left(1-2 \phi_{y x}\right) \operatorname{Var}\left(b_{x}\right)+G_{y y}^{2} \phi_{x y}\left(1-2 \phi_{x y}\right) \operatorname{Var}\left(b_{y}\right)+\mathcal{V}\right]
\end{gathered}
$$

407 which corresponds to a relationship with $\phi$ as in Figs. 4F, S1F, and S2F. This analysis

408 illustrates that reciprocal IIGEs have greatest impact on mediating coevolutionary outcomes

409 in a trait-matching models when IIGEs are similar in both sign and magnitude. Biologically, 
410

411

412

413

414

415

416

417

418

419

420

421

422

423

424

425

$$
\begin{aligned}
& \beta_{x x}=-\beta_{x y}=-2 b_{x y}\left(\bar{z}_{x}-\bar{z}_{y}\right) \\
& \beta_{y y}=-\beta_{y x}=-2 b_{y x}\left(\bar{z}_{y}-\bar{z}_{x}\right)
\end{aligned}
$$

$$
\begin{aligned}
& w_{x}=\alpha_{x}-b_{x y}\left(\mathrm{z}_{x}-\mathrm{z}_{y}\right)^{2}+\varepsilon_{x} \\
& w_{y}=\alpha_{y}-b_{y x}\left(\mathrm{z}_{y}-\mathrm{z}_{x}\right)^{2}+\varepsilon_{y} .
\end{aligned}
$$

427 In the simplest case of no IIGEs, $G_{x y}=0$, and no variation in $b$, these gradients yield

428

$$
\operatorname{Cov}\left(\Delta \bar{z}_{x}, \Delta \bar{z}_{y}\right)=-4 G_{x x} G_{y y} b_{x y} b_{y x} \operatorname{Var}\left(\bar{z}_{x}-\bar{z}_{y}\right)
$$


430 Thus, within the limits of genetic variation and ignoring stochastic forces (as we have done),

431 evolutionary response will be perfectly correlated in the phenotypic matching model. When

$432 b_{x y}$ and $b_{y x}$ share the same sign, this covariance in evolutionary response will be negative

433 because each species will be evolving towards (or away) from each other in trait space. This

434 covariance in evolutionary response occurs even without variation in $b$ or covariance in trait

435 means. These results are broadly consistent with other analyses of the trait matching model,

436 which indicate that this model can generate relatively strong covariance in phenotypic means

437 across populations (Nuismer et al. 2010). Adding indirect genetic effects and assuming

$G_{x y}=0$ and noting that $\operatorname{Var}\left(\bar{z}_{x}-\bar{z}_{y}\right)=\operatorname{Var}\left(\bar{z}_{y}-\bar{z}_{x}\right)$,

439

$$
\begin{gathered}
\operatorname{Cov}\left(\Delta \bar{z}_{x}, \Delta \bar{z}_{y}\right)=\mathcal{U}\left[-4 b_{x y} b_{y x} G_{x x} G_{y y} \operatorname{Var}\left(\bar{z}_{x}-\bar{z}_{y}\right)+\right. \\
4 b_{x y}{ }^{2} G_{x x}^{2} \phi_{y x} \operatorname{Var}\left(\bar{z}_{x}-\bar{z}_{y}\right)+4 b_{y x}{ }^{2} G_{y y}^{2} \phi_{x y} \operatorname{Var}\left(\bar{z}_{x}-\bar{z}_{y}\right) \\
-8 b_{x y} b_{y x} G_{x x} G_{y y} \phi_{x y} \phi_{y x} \operatorname{Var}\left(\bar{z}_{x}-\bar{z}_{y}\right)+ \\
4 b_{x y} b_{y x} G_{x x} G_{y y} \phi_{x y} \operatorname{Var}\left(\bar{z}_{x}-\bar{z}_{y}\right)+4 b_{y x} b_{x y} G_{x x} G_{y y} \phi_{y x} \operatorname{Var}\left(\bar{z}_{x}-\bar{z}_{y}\right) \\
\left.-8 b_{x y}{ }^{2} G_{x x}^{2} \phi_{y x}^{2} \operatorname{Var}\left(\bar{z}_{x}-\bar{z}_{y}\right)-8 b_{y x}{ }^{2} G_{y y}^{2} \phi_{x y}^{2} \operatorname{Var}\left(\bar{z}_{x}-\bar{z}_{y}\right)+\mathcal{V}\right]
\end{gathered}
$$

440 This expression again shows the covariance in evolutionary response occurs even in the

441 absence of (co)variance in $b$, and the addition of IIGEs alter the magnitude of the covariance

442 substantially. Analysis of more complex cases of the trait matching model, for example

443 where selection varies across space and/or across terms in the expanded polynomial

$444 \quad\left(z_{x}-z_{y}\right)^{2}$, would be straightforward in this framework.

As a final example of how this approach can be used to understand simple variations 
447 where fitness depends on the interaction between traits of the two species. Consider a fitness

448 model where relative fitness depends solely on the product of the two phenotypes:

449

$$
\begin{aligned}
& w_{x}=\alpha_{x}+b_{x y} z_{x} z_{y}+\varepsilon_{x} \\
& w_{y}=\alpha_{y}+b_{y x} z_{y} z_{x}+\varepsilon_{y} .
\end{aligned}
$$

450

451 This model is conceptually and mathematically similar to the previous trait matching model,

452 but importantly lacks stabilizing selection terms (e.g., $\left.b_{x y} z_{x}^{2}\right)$ present in the trait matching

453 model. Because of this, directional natural selection depends only on the means of the other

454 species:

455

$$
\begin{aligned}
& \beta_{x x}=b_{x y} \bar{z}_{y} \\
& \beta_{x y}=b_{x y} \bar{z}_{x} \\
& \beta_{y y}=b_{y x} \bar{z}_{x} \\
& \beta_{y x}=b_{y x} \bar{z}_{y}
\end{aligned}
$$

456

457 This relationship may cause selection to (co)vary across populations even when $b_{x y}$ and $b_{y x}$ are homogeneous. In the absence of IIGEs, this simplest case would lead to a coevolutionary covariance defined by

460

$$
\operatorname{Cov}\left(\Delta \bar{z}_{x}, \Delta \bar{z}_{y}\right)=G_{x x} G_{y y} b_{x y} b_{y x} \operatorname{Cov}\left(\bar{z}_{x}, \bar{z}_{y}\right) .
$$


461 Thus, any initial covariance in the population means leads to a covariance in the response to

$$
\begin{gathered}
\operatorname{Cov}\left(\Delta \bar{z}_{x}, \Delta \bar{z}_{y}\right)=\mathcal{U}\left[G_{x x} G_{y y} b_{x y} b_{y x} \operatorname{Cov}\left(\bar{z}_{x}, \bar{z}_{y}\right)\right. \\
+G_{x x}^{2} \phi_{y x} b_{x y}^{2} \operatorname{Var}\left(\bar{z}_{y}\right)+G_{y y}^{2} \phi_{x y} b_{y x}^{2} \operatorname{Var}\left(\bar{z}_{x}\right)+ \\
2 G_{x x} G_{y y} \phi_{x y} \phi_{y x} b_{x y} b_{y x} \operatorname{Cov}\left(\bar{z}_{x}, \bar{z}_{y}\right) \\
+G_{x x} G_{y y} \phi_{x y} b_{x y} b_{y x} \operatorname{Var}\left(\bar{z}_{y}\right)+G_{x x} y G_{y} \phi_{y x} b_{x y} b_{y x} \operatorname{Var}\left(\bar{z}_{x}\right)+ \\
\left.2 G_{x x}^{2} \phi_{y x}^{2} b_{x y}^{2} \operatorname{Cov}\left(\bar{z}_{x}, \bar{z}_{y}\right)+2 G_{y y}^{2} \phi_{x y}^{2} b_{y x}^{2} \operatorname{Cov}\left(\bar{z}_{x}, \bar{z}_{y}\right)+\mathcal{V}\right] .
\end{gathered}
$$

465

466 This equation again shows that when IIGEs are present, any variance across populations in the trait mean of either species leads to a cross-species covariance in the response to selection (terms 2, 3, 5, and 6). Cross species covariance in selection response is also mediated further by covariance in trait means in this model (terms 1, 4, 7, and 8) as well as higher order products of IIGEs captured in $V$ (equation 11). These effects may lead trait means to become correlated across populations in future generations. This analysis, particularly contrasting equations 16 vs. 20 and 17 vs. 21, also reveals that coevolutionary dynamics can be substantially different even across models that share a common polynomial relative fitness

474 function.

Discussion

477 Our model adapts the theory of trait-based intraspecific social evolution to the phenotypic

478 interface between two coevolving species. We show that two forms of interspecific

479 interaction, interspecific indirect genetic effects (IIGEs) and cross-species selection 480 (analogous to within-species social selection), both contribute to correlated evolution 
between interacting species. Our analysis shows that reciprocal IIGEs modulate selection can generate a coevolutionary covariance even in the absence of covariance in selection, or even the absence of genetic variance in one species. When selection does covary between species across populations, reciprocal IIGEs will promote changes in the magnitude of coevolution and even reversals in the expected among-population covariance. IIGEs also allow cross-species selection, which we model as the effect of the traits of one species on the fitness of another, to influence evolutionary response. Such a response may also be mediated by cross-species genetic assortment between interacting individuals. When IIGEs and crossspecies selection act together, effects on the coevolutionary covariance can be complex, with dramatic changes in the expected sign and magnitude of correlated evolution occurring with subtle changes in these parameters of interspecific social interaction. Our results indicate that whenever coevolving species socially interact to modify expression of one another's phenotypes, these interspecific social interactions are key to understanding coevolution. the phenotypic value of the interspecific individual(s) with which an organism interacts. To our knowledge, this type of environmental effect on between-species coevolution has been considered in only two other theoretical studies (Scheiner et al. 2015, O'Brien et al. 2021; but see Shuster et al. 2006, Witham et al. 2020 for a variance-partitioning approach). Scheiner et al. (2015) consider a special case of our model, where evolvable IIGEs are present in only

504 one of the two interacting species. In this non-reciprocal model, they show a much more 505 limited role for IIGEs in coevolution. Our results are broadly consistent with this conclusion, 
in that IIGEs in only a single species do not generate the reciprocal effects that lead to

507 massive inflation of evolutionary response. However, IIGEs in only a single species (e.g.,

$\left.\boldsymbol{\Phi}_{x y}=\mathbf{0}, \boldsymbol{\Phi}_{y x} \neq \mathbf{0}\right)$ still play a role in mediating response to interspecific social selection

whenever interspecific social selection is a function of individual trait values (as opposed to

510 the population mean, as modeled by Scheiner et al. 2015). We also note that our fully

511 multivariate model accommodates the possibility that reciprocal IIGEs act across different

512 types or numbers of traits in the two interacting species. More recently, O'Brien et al. (2021;

513 see also Queller 2014) developed a model of coevolution between host plant and microbial

514 symbionts. Their parameterization differed from ours in that they consider evolution of a

515 single joint trait governed by genetic variation in host and symbiont, and so is most directly

516 applicable to plant-microbe systems or other intimate interactions. Nonetheless, their model

517 shows an important role for reciprocal fitness feedbacks, consistent with the conclusions of

518 our trait-based model.

Within-species models of interacting phenotypes clarify the line between genetic and

environmental effects, furthering an understanding of how genotypes expressed in an

individual that act as environments for other individuals can influence phenotypic change

522 (Wolf et al. 1998, Wolf 2003). That is, IGEs are genetically-based environments that

523 influence phenotypic expression during interactions. Conceptually, this relationship is similar to genetically based plasticity. Extending these types of models to the case of interspecific interaction carries similar challenges and benefits. We have referred to reciprocal change in phenotypic means between interacting species as "coevolution," even when these effects are mediated by IIGEs. This is a broad use of the term coevolution, as IIGEs are an environmental effect that can themselves evolve, again similar in concept to phenotypic plasticity. However, a critical difference is that changes in phenotypic response mediated by 
4). Our point is not to broaden the definition of coevolution, but rather to highlight that

532 IIGEs can have substantial impact on patterns of phenotypic divergence in coevolving

533 species. For example, our model highlights that genetic divergence across populations of a

534 single species is sufficient to generate tightly-coupled patterns of correlated change in an

535 interacting species, a result that suggests the challenges of interpreting correlated phenotypes

536 as evidence of genetic response to reciprocal selection may be even greater than already

537 appreciated (e.g. Nuismer et al. 2010, Gomulkiewicz et al 2007, Janzen 1980).

538 Interspecific indirect genetic effects, or at least the potential for a prevalence of such

539 effects, appear to be commonplace in many biological systems. In Table 1, we provide in a

540 breakdown of types of biological interaction in which there is a large literature suggesting

541 importance of IIGE-like phenomena. These types of effects on trait expression across

542 species, widely appreciated in their own specific contexts (Weis and Abrahamson 1986,

543 Peacor and Werner 2001, Werner and Peacor 2003, Chen 2008, Thomas et al. 2012, O'Brien

544 et al. 2021), have taken on a variety of different forms. We suggest that these disparate

545 biological phenomena may nonetheless share a commonality-reciprocal effects on trait

546 expression across interspecific partners - that we have shown can affect the coevolutionary

547 process in dramatic, and in some cases predictable, ways.

548 Cross-species selection features prominently in verbal descriptions of the

549 coevolutionary process (Thompson 1982), and we show that such selection is especially

550 important in the presence of interspecific indirect genetic effects. When individual trait

551 values of one species affect individual fitness of another, focal species, this cross-species

552 selection can manifest evolutionary change in the focal species when there is phenotypic

553 assortment between interspecific interactants. This assortment, analogous to that required for

554 evolutionary response to social selection within species (Wolf et al. 1999, McGlothlin et al.

555 2010, Brodie et al. submitted), can be generated directly by a non-random genetic assortment, 
or via IIGEs. Examples of processes that could generate direct genetic assortment between

557 interacting individuals of two different species include shared genetic structure, to the extent

558 that such structure manifests assortment of breeding values for the relevant traits. Such

559 shared genetic structure could arise through shared features of the environment that limit

560 gene flow and panmictic mating in both species, or alternatively, through variation in habitat

561 preference across individuals of both species. Direct genetic assortment could also arise

562 through behavioral preference for certain trait values in heterospecific partners. Such

563 preferences may be especially common in predator-prey interactions, where, for example,

564 predator body size may be expected to coevolve with behavioral preference for prey size

565 (Troost et al. 2008). Currently, it is unclear how common this type of cross-species genetic

566 assortment may be, although in part this likely reflects a lack of studies that have attempted to

567 measure assortment between breeding values of individuals of separate species. Moreover,

568 when it does occur, such assortment is likely to be transient because it does not rely on

569 transmission of pleiotropic alleles that may stabilize within-species genetic correlations over

570 multiple generations. The substantial evidence for IIGEs, but limited evidence of $G_{x y}$

571 suggests that IIGEs may play a prominent role in mediating any realized response to cross-

572 species selection.

573 Our results also indicate that nonlinear effects on cross-species selection can

574 contribute to coevolution even in the absence of genetic assortment or IIGEs. This form of

575 selection corresponds to an interaction between focal and interspecific-partner trait values for

576 focal individual fitness. The effect of this form of cross-species selection on evolutionary

577 response in a population depends on the mean genotype of the other species, and thus

578 represents a diffuse effect of population mean phenotype of the coevolving species. Such

579 interspecific interactions are potentially less intimate, for example diffuse predator chemical

580 cues in aquatic environments, than the individual level interactions (e.g., of host and parasite) 
581

582

583

584

585

586

587

588

589

590

591

592

593

594

595

596

597

598

599

600

601

602

603

604

605

required to generate response from linear cross-species selection. Across populations,

nonlinear cross-species selection contributes to coevolution via covariance in mean genetic

values and/or linear selection between the species. This result is consistent with past models

of coevolution, verbal and mathematical, that indicate trait interactions for fitness are a key

feature of coevolution (Thompson 1982, 1994, 2005, Nuismer 2017), and in our model, such

interactions lead to a dependence between selection in one species and the mean trait value of

another. By defining these interaction terms in the framework of social evolution, our model

adds to past work by indicating that reciprocal IIGEs can substantially increase the degree to

which trait interactions for fitness contribute to reciprocal evolutionary change.

A key feature of our model is the development of a formal expression for the expected

covariance in evolutionary response, $\operatorname{Cov}\left(\Delta \bar{z}_{x}, \Delta \bar{z}_{y}\right)$. This coevolutionary covariance is

expected to be a key contributor to generating among-population covariation in species mean

trait values, a major focus in many empirical (Thompson 1994, 1995, Zangerl and

Berenbaum 2003, Toju and Sota 2005, Hanifin et al. 2008, Hague et al. 2020) and theoretical

(Nuismer et al. 2010, Nuismer and Week 2019, Week and Nuismer 2019) studies of

coevolution. Importantly, similar to existing within-species models of among-population

quantitative genetic variation (Zeng 1988, Chenoweth et al. 2010), defining this

coevolutionary covariance illustrates how selection, IIGEs, and genetics may contribute to

patterns of trait variation across populations.

Our model subsumes mechanistic detail into broad statistical descriptions of species

interactions and thus provides a general description of how IIGEs and cross-species selection,

when present, contribute to reciprocal evolutionary change and correlated evolution across

populations. In contrast to our approach, some models of coevolution have focused instead

on specific ecological mechanisms that generate trait-fitness relationships between interacting

species (reviewed in Nuismer 2017). By highlighting the key parameters that contribute to 
606

607

608

609

610

611

612

613

614

615

616

617

618

619

620

621

622

623

624

625

626

627

628

629

630

coevolution-covariance in natural selection, covariance in cross-species selection, and

IIGEs - our model indicates various pathways through which specific ecological mechanisms may affect coevolution. Our framework can be tailored to specific scenarios by substituting different fitness models into the general equations we present here.

Our model generates quantitative predictions for the shape of coevolution that are directly testable with empirical data because it focuses on estimable statistical effects of underlying ecological mechanisms rather than the mechanisms themselves, which are often unknown (Wade and Kalisz 1990). For example, using an empirical estimate of $\boldsymbol{\Phi}$ (which could be measured using methods analogous to those used to measure within-species indirect genetic effects; Bleakley and Brodie 2009, McGlothlin and Brodie 2009), one could use matrix comparison of covariances among population means and the covariance terms presented here to quantitatively test the contribution of IIGEs to among-population covariance in selection response between two interacting species (e.g., see Chenoweth et al. 2010 for a within-species test of the predictions of Zeng's 1988 model). More generally, Week and Nuismer (2019; see also Nuismer and Week 2019) have shown how datasets of among-population variation in trait means can be used to test for conformation to expectations from coevolutionary models. Concomitantly, our models show how environmental effects can be partitioned into terms describing genotypes of other species in the ecological community, which could be useful in understanding when and why evolutionary response fails to conform to predictions arising from the standard breeder's equation.

Social interactions between individuals of the same species play a central role in the evolutionary process. Within a single lineage, indirect genetic effects and social selection fundamentally change selection response, the expression of genetic variance, and together determine the course of social evolution (Moore et al. 1997, Wolf et al. 1998, Wolf et al. 
bioRxiv preprint doi: https://doi.org/10.1101/2021.04.14.439826; this version posted September 8, 2021. The copyright holder for this preprint (which was not certified by peer review) is the author/funder, who has granted bioRxiv a license to display the preprint in perpetuity. It is made available under aCC-BY 4.0 International license.

631 1999, McGlothlin et al. 2010). We have shown that these effects of interactions among

632 individuals may transcend species boundaries and profoundly impact the dynamics of

633 coevolution between interacting lineages. 


\section{Literature Cited}

635

636

637

638

639

640

641

642

643

644

645

646

647

648

649

650

651

652

653

654

655

656

657

658

659

660

661

662

663

664

665

666

667

668

669

670

671

672

673

674

675

676

677

678

679

680

681

682

Abrahamson, W. G., and A. E. Weis. 1997. Evolutionary Ecology Across Three Trophic Levels: Goldenrods, Gallmakers, and Natural Enemies. Princeton University Press Princeton.

Abrams, P. A., Y. Harada, and H. Matsuda. 1993. On the relationship between quantitative genetic and ESS models. Evolution 47:982-985.

Agrawal, A. A. 2001. Phenotypic plasticity in the interactions and evolution of species. Science 294:321-326.

Arnold, S. J., M. E. Pfrender, and A. G. Jones. 2001. The adaptive landscape as a conceptual bridge between micro- and macroevolution. Genetica 112/113:9-32.

Arnqvist, G., and L. Rowe. 2002. Antagonistic coevolution between the sexes in a group of insects. Nature 415:787-789.

Barribeau, S. M. 2014. Gene expression differences underlying genotype-by-genotype specificty in a host-parasite system. Proceedings of the National Academy of Sciences 111:3496-3501.

Bijma, P., and M. J. Wade. 2008. The joint effects of kin, multilevel selection and indirect genetic effects on response to genetic selection. Journal of Evolutionary Biology 21:1175-1188.

Bleakley, B. H., and E. D. Brodie III. 2009. Indirect genetic effects influence antipredator behavior in guppies: Estimates of the coefficient of interaction $p s i$ and the inheritance of reciprocity. Evolution 63:1796-1806.

Boersma, M., P. Spaak, and L. De Meester. 1998. Predator-mediated plasticity in morphology, life history, and behavior of Daphnia: the uncoupling of responses. American Naturalist 152:237-248.

Borell, E. M., A. Foggo, and R. A. Coleman. 2004. Induced resistance in intertidal macroalgae modifies feeding behavior of herbivorous snails. Oecologia 140:328-334.

Brodie, E. D., III, and E. D. Brodie, Jr. 1999. Predator-prey arms races. BioScience 49:557568.

Brodie, E. D. Jr., B. J. Ridenhour, and E. D. Brodie III. 2002. The evolutionary response of predators to dangerous prey: hotspots and coldspots in the geographic mosaic of coevolution between garters snakes and newts Evolution 56:2067-2082.

Brodie, E. D., III and B. J. Ridenhour 2003 Reciprocal selection at the phenotypic interface of coevolution. Integrative and Comparative Biology 43:408-418

Brodie, E.D., III, P. A. Cook, R. A. Costello, V. A. Formica. Submitted. Phenotypic assortment changes the landscape of selection. Journal of Heredity.

Chen, M.-S. 2008. Inducible direct plant defense against insect herbivoes: A review. Insect Science 15:101-114.

Chenoweth, S. F., H. D. Rundle, and M. W. Blows. 2010. The contribution of selection and genetic contraints to phenotypic divergence. American Naturalist 175:186-196.

Damian, R. T. 1997. Parasite immune evasion and exploitation: reflections and projections. Parasitology 115:S169-175.

Dawkins, R. 1982. The Extended Phenotype. Oxford Univ. Press, Oxford.

Drown, D. M., and M. J. Wade. 2014. Runaway coevolution: adaptation to heritable and nonheritable environments. Evolution 68:3039-3046.

Dybdahl, M. F., C. E. Jenkins, and S. L. Nuismer. 2014. Identifying the molecular basis of host-parasite coevolution: merging models and mechanisms. American Naturalist 184:1-13.

Ehrlich, P. R., and P. H. Raven. 1964. Butterflies and plants: a study in coevolution. Evolution 18:586-608. 
Fisher, D.N. and A. McAdam. 2019. Indirect genetic effects clarify how traits can evolve even when fitness does not. Evolution Letters 3:4-14.

Foster, K. R., J. Schluter, K. Z. Coyte, and S. Rakoff-Nahoum. 2017. The evolution of the host microbiome as an ecosystem on a leash. Nature 548:43-51.

Friesen, M. L., S. S. Porter, S. C. Stark, E. J. von Wettberg, J. L. Sachs, and E. MartinezRomero. 2011. Microbially mediated plant functional traits. Annual Review of Ecology and Systematics 42:23-46.

Fuess, L., J. N. Weber, S. den Haan, N. C. Steinel, K. C. Shim, and D. I. Bolnick. 2020. A test of the Baldwin effect: Differences in both constitutive expression and inducible responses to parasites underlie variation in host response to a parasite. bioRxiv https://doi.org/10.1101/2020.07.29.216531.

Gianinazzi-Pearson, V., N. Séjalon-Delmas, A. Genre, S. Jeandroz, and P. Bonfante. 2007. Plants and arbuscular mycorrhizal fungi: cues and communication in the early steps of symbiotic interactions. Advances in Botanical Research 46:181-219.

Gomulkiewicz, R., D.M. Drown, M.F. Dybdahl, W. Godsoe, S.L. Nuismer, K.M. Pepin, B.J. Ridenhour, C.I. Smith, and J.B. Yoder. 2007. Dos and don'ts of testing the geographic mosaic theory of coevolution. Heredity 98:249-258.

Hague, M. T. J., A. N. Stokes, C. R. Feldman, E. D. Brodie Jr., and E. D. Brodie III. 2020. The geographic mosaic of arms race coevolution is closely matched to prey population structure. Evolution Letters 164:1567-16.

Hanifin, C. T., E. D. Brodie Jr., and E. D. Brodie III. 2008. Phenotypic mismatches reveal escape from arms-race coevolution. PLoS Biology 6:e60.

Janzen, D. H. 1980. When is it coevolution? Evolution 34:611-612.

Johnson, S. D., and B. Anderson. 2010. Coevolution between food-rewarding flowers and their pollinators. Evolution: Eduacation and Outreach 3:32-39.

Kirkpatrick, M. and R. Lande 1989. The evolution of maternal characters. Evolution 43:485503.

Korgaonkar, A., C. Han, A. L. Lemire, I. Siwanowicz, D. Bennouna, R. E. Kopec, P. Andolfatto, S. Shigenobu, and D.L. Stern. 2021. A novel family of secreted insect proteins linked to plant gall development. Current Biology 31:1-14

Lande, R. 1979. Quantitative genetic analysis of multivariate evolution, applied to brain: body size allometry. Evolution 33:402-416.

Lande, R., and S. J. Arnold. 1983. The measurement of selection on correlated characters. Evolution 37:1210-1226.

McAdam, A.G., S. Boutin, D. Réale, and D. Berteaux. 2002. Maternal effects and the potential for evolution in a natural population of animals. Evolution 56: 846-851.

McGlothlin, J. W., and E. D. Brodie III. 2009. How to measure indirect genetic effects: the congruence of trait-based and variance-partitioning approaches. Evolution 63:17851795.

McGlothlin, J. W., E. Akçay, E. D. Brodie III, A. J. Moore, and J. Van Cleve. 2021. A synthesis of game theory and quantitative genetic models of social evolution. bioRxiv https://doi.org/10.1101/2021.03.27.437341

McGlothlin, J. W., A. J. Moore, J. B. Wolf, and E. D. Brodie III. 2010. Interacting phenotypes and the evolutionary process. III. Social evolution. Evolution 64:25582574.

Moore, A. J., E. D. Brodie III, and J. B. Wolf. 1997. Interacting phenotypes and the evolutionary process: I. Direct and indirect genetic effects of social interactions. Evolution 51:1352-1362.

Mousseau, T.A. and C.W. Fox. 1998. The adaptive significance of maternal effects. Trends in Ecology and Evolution 13:403-407 
Nuismer, S. L. 2017. Introduction to Coevolutionary Theory. W.H. Freeman and Company New York

Nuismer, S. L., B. J. Ridenhour, and B. P. Oswald. 2007. Antagonistic coevolution mediated by phenotypic differences between quantitative traits. Evolution 61:1823-1834.

Nuismer, S.L., R. Gomulkiewicz, and B.J. Ridenhour. 2010. When is correlation coevolution. The American Naturalist 175:525-537.

Nuismer, S.L., and B. Week. 2019. Approximate Bayesian estimation of coevolutionary arms races. PLoS Computational Biology 15:e1006988.

Ohgushi, T. 2005. Indirect interaction webs: Herbivore-induced effects through trait change in plants Annual Review of Ecology, Evolution, and Systematics 36:81-105.

Oladiran, A., and M. Belosevic. 2012. Immune evasion strategies of trypanosomes: a review. Journal of Parasitology 98:284-292.

O’Brien, A. M., C. N. Jack, M. L. Friesen, and M. E. Frederickson. 2021. Whose trait is it anyways? Coevolution of joint phenotypes and genetic architecture in mutualisms. Proceedings of the Royal Society of London Series B. 288:20202483.

Peacor, S. D., and E. E. Werner. 2001. The contribution of trait-mediated indirect effects to the net effects of a predator. Proceedings of the National Academy of Sciences 98:3904-3908.

Pellmyr, O. 2003. Yuccas, yucca moths, and coevolution: A review. Annals of the Missouri Botanical Garden 90:35-55.

Phillips, P.C., and S.J. Arnold. 1989. Visualizing multivariate selection. Evolution 43:12091222.

Preisser, E. L., D. I. Bolnick, and M. F. Benard. 2005. Scared to death? The effects of intimidation and consumption in predator-prey interactions. Ecology 86:501-509.

Price, G. R. 1970. Selection and covariance. Nature 227:520-521.

Price, G. R. 1972. Extension of covariance mathematics. Annals of Human Genetics 35:485490.

Queller, D. C. 2014. Joint phenotypes, evolutionary conflict and the fundamental theorem of natural selection. Philosophical Transactions of the Royal Society B. 369:20130423.

Ridenhour, B. J. 2005. Identification of selective sources: Partitioning selection based on interactions. The American Naturalist 166:12-25.

Robertson, A. 1966. A mathematical model of the culling process in dairy cattle. Animal Production 8:95-108.

Scharsack, J. P., A. Gossens, F. Franke, and J. Kurtz. 2013. Excretory products of the cestode, Schistocephalus solidus, modulate in vitro responses of leukocytes from its specific host, the three-spined stickleback (Gasterosteus aculeatus). Fish and Shellfish Immunology 35:1779-1787.

Scharsack, J. P., M. Kalbe, R. Derner, J. Kurtz, and M. Milinski. 2004. Modulation of granulocyte responses in three-spined sticlebacks Gasterosteus aculeatus infected with the tapeworm Schistocephalus solidus. Diseases of Aquatic Organisms 59:141-150.

Scharsack, J. P., K. Koch, and K. Hammerschmidt. 2007. Who is in control of the stickleback immune system: interactions between Schistocephalus solidus and its specific vertebrate host. Proceedings of the Royal Society of London Series B. 274:31513158.

Scheiner, S. M., R. Gomulkiewicz, and R. D. Holt. 2015. The genetics of phenotypic plasticity. XIV. Coevolution. American Naturalist 185:594-609.

Schmid-Hempel, P. 2008. Parasite immune evasion: a momentous molecular war. Trends in Ecology and Evolution 23:318-326. 
Shuster, S. M., E. V. Lonsdorf, G. M. Wimp, J. K. Bailey, and T. G. Whitham. 2006. Community heritability measures the evolutionary consequences of indirect genetic effects on community structure. Evolution 60:991-1003.

Siepielski, A. M., K. M. Gotanda, M. B. Morrissey, S. E. Diamond, J. D. DiBattista, and S. M. Carlson. 2013. The spatial patterns of directional phenotypic selection. Ecology Letters 16:1382-1392.

Stappenbeck, T. S., and H. W. Virgin. 2016. Accounting for reciprocal host-microbiome interactions in experimental science. Nature 534:191-199.

Stearns, S. C. 2012. Evolutionary routes leading to host manipulation by parasites: Afterward in F. Thomas, J. Brodeur, and D. P. Hughes, editors. Host manipulation by parasites. Oxford University Press, Oxford.

Tams, V., J. H. Nickel, A. Ehring, and M. Cordellier. 2019. Insights into the genetic basis of predator-induced response in Daphnia - a comparative transcriptomic approach. bioRxiv. https://doi.org/10.1101/503904

Thomas, F., J. Brodeur, and D. P. Hughes. 2012. Host manipulation by parasites Oxford University Press, Oxford.

Thompson, J. N. 1982. Interaction and Coevolution. University of Chicago Press, Chicago.

Thompson, J. N. 1994. The Coevolutionary Process. University of Chicago Press, Chicago.

Thompson, J. N. 2005. The Geographic Mosaic of Coevolution. University of Chicago Press, Chicago.

Toju, H., and T. Sota. 2005. Imbalance of predator and prey armament: geographic clines in phenotypic interface and natural selection. American Naturalist 167:105-117

Troost, T. A., B. W. Kooi, and U. Dieckmann. 2008. Joint evolution of predator body size and prey size preference. Evolutionary Ecology 22:771-799.

Urban, M.C. 2011. The evolution of species interactions across natural landscapes. Ecology Letters 14:723-732.

Wade, M. J., and Kalisz. 1990. The causes of natural selection. Evolution 44:1947-1955.

Walsh, M. R., and D. M. Post. 2011. The impact of intraspecific vriation in a fish predator on the evolution of phenotypic plasticity and investment in sex in Daphnia ambigua. Journal of Evolutionary Biology 25:80-89.

Weber, A., and S. Declerck. 1997. Phenotypic plasticity of Daphnia life history traits in response to predator kairomones: genetic variability and evolutionary potential Hydrobiologia 360:89-99.

Weber, J. N., N. C. Steinel, K. C. Shim, and D. I. Bolnick. 2017. Recent evolution of extreme cestode growth suppression by a vertebrate host. Proceedings of the National Academy of Sciences 114:6575-6580.

Week, B. and S.L. Nuismer. 2019. The measurement of coevolution in the wild. Ecology Letters 22:717-725

Weis, A. E., and W. G. Abrahamson. 1986. Evolution of host-plant manipulations by gall makers: ecological and genetic factors in the Solidago-Eurosta system. American Naturalist 127:681-695.

Weis, A.E., W.G. Abrahamson, and M.C. Anderson. 1992. Variable selection on Eurosta's gall size, I: The extent and nature of variation in phenotypic selection. Evolution 46:1674-1697.

Werner, E. E., and S. D. Peacor. 2003. A review of trait-mediated indirect interactions in ecological communities. Ecology 84:1083-1100.

West-Eberhard, M. J. 1979. Sexual selection, social competition, and evolution. Proceedings of the American Philosophical Society 123:222-234.

West-Eberhard, M. J. 1983. Sexual selection, social competition, and speciation. Quarterly Review of Biology 58:155-183. 
831

832

833

834

835

836

837

838

839

840

841

842

843

844

845

846

847

848

849

850

851
West-Eberhard, M. J. 1984. Sexual selection, competitive communication and speciesspecific signals in insects. Pages 283-342 in T. Lewis, editor. Insect communication. Academic Press, New York.

Whitham, T. H., G. J. Allan, H. F. Cooper, and S. M. Shuster. 2020. Intraspecific genetic variation and species interactions contribute to community evolution. Annual Review of Ecology and Systematics 51:587-612.

Wolf, J. B., E. D. Brodie III, J. M. Cheverud, A. J. Moore, and M. J. Wade. 1998. Evolutionary consequences of indirect genetic effects. Trends in Ecology and Evolution 13:64-69.

Wolf, J. B., E. D. Brodie III, and A. J. Moore. 1999. Interacting phenotypes and the evolutionary process. II. Selection resulting from social interactions. American Naturalist 153:254-266.

Wolf, J.B. 2003. Genetic architecture and evolutionary constraint when the environment contains genes. Proceedings of the National Academy of Sciences 100:4655-4660.

Zangerl, A. R., and M. R. Berenbaum. 2003. Phenotype matching in wild parsnip and parsnip webworms: causes and consequences. Evolution 57:806-815

Zeng, Z.-B. 1988. Long-term correlated response, interpopulation covariation, and interspecific allometry. Evolution 42 363-374.

Züst, T., S. Mou, and A.A. Agrawal. 2018. What doesn't kill you makes you stronger: The burdens and benefits of toxin sequestration in a milkweed aphid. Functional Ecology 8:1972-1981 


\section{Supplemental Figures}
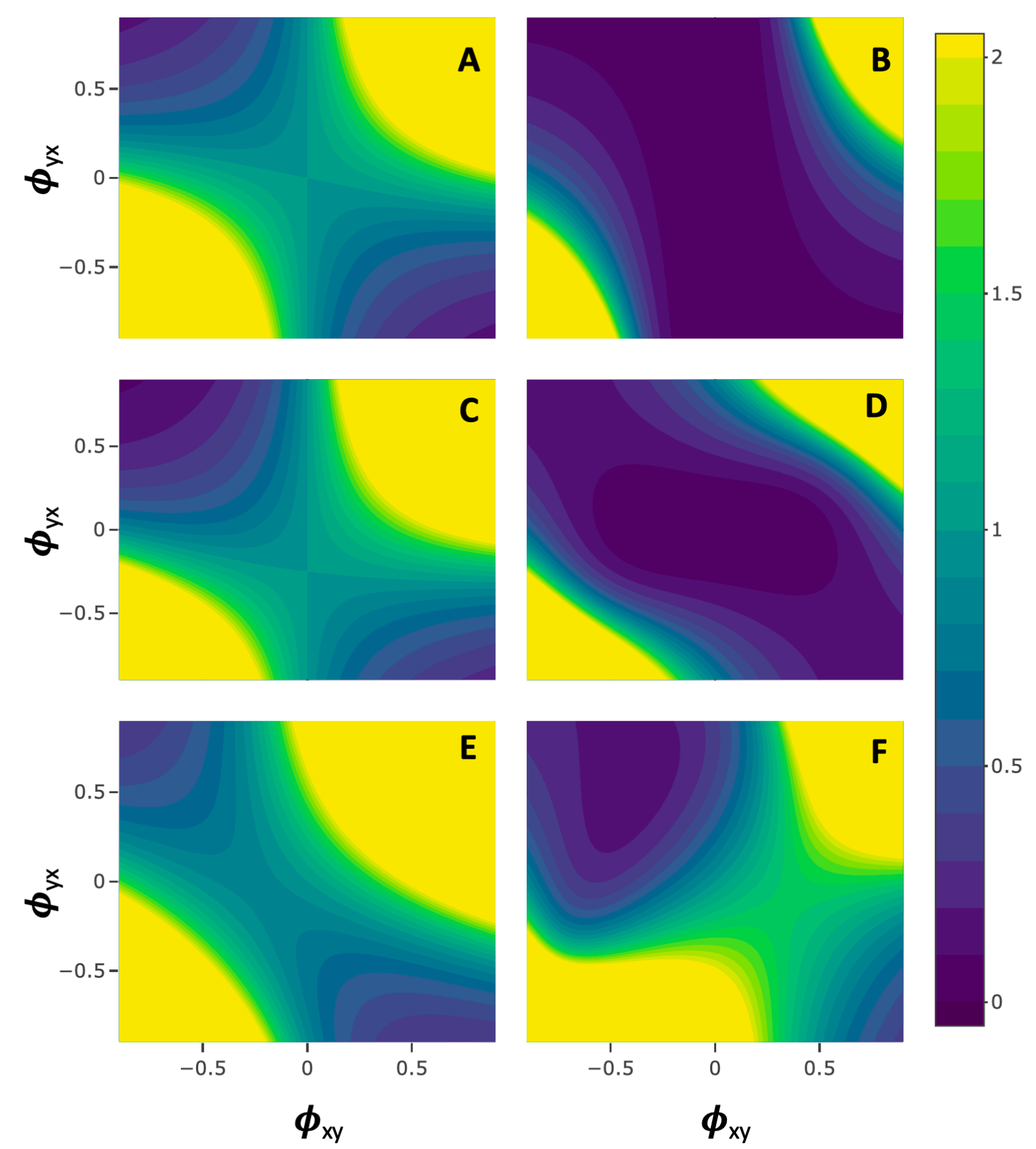

Figure S1. IIGEs accelerate evolutionary rate in a single species. Panels show the evolutionary rate, $\operatorname{Var}\left(\Delta \overline{\mathrm{Z}}_{\mathrm{x}}\right)$, of species $x$ as a function of the indirect genetic effect parameters $\phi$, under the same parameter values as in Figure 3. Reciprocal IIGEs between interacting species generally accelerate evolutionary rate. Note that in the absence of any other effects, the evolutionary rate is equal to the variance in natural selection, which is unity in Panels $\mathrm{A}, \mathrm{C}, \mathrm{E}$, and $\mathrm{F}$. In panel $\mathrm{D}$, evolutionary rate is driven entirely by cross-species selection and IIGEs. In panel $\mathrm{B}$, where $\mathrm{G}_{x x}=0$, evolutionary rate in species $x$ is driven entirely by reciprocal IIGEs and evolutionary change in species $y$. 



Figure S2. Among population covariances. Panels show the coevolutionary covariance between two interacting species as a function of the indirect genetic effect parameters $\phi$, from equation (7) unstandardized. Parameter values are as in Figs. 4 and S1. For all panels, genetic variances were set to unity. 


\section{Appendix}

\section{A1. Phenotypic covariance between interacting species}

854 For a single trait, we can solve for the covariance between $z_{y}$ and $z_{x}$ to partition the

855 phenotypic covariance between individuals of two interacting species into terms describing

856 the contribution of IIGEs and terms describing non-random genetic assortment. Assuming

$$
\begin{gathered}
C_{x y}=\left(1-\phi_{y x} \phi_{x y}\right)^{-2}\left[\left(1+\phi_{y x} \phi_{x y}\right) G_{x y}+\phi_{y x}\left(G_{x}+E_{x}\right)+\phi_{x y}\left(G_{y y}\right.\right. \\
\left.\left.+E_{x}\right)\right]
\end{gathered}
$$

where $E_{x}$ and $E_{y}$ represent within-species environmental variance and $G_{x y}=G_{y x}$. When

IIGEs are absent, non-random genetic assortment $G_{x y}$ is the sole contributor to the

phenotypic association between individuals of coevolving species. When IIGEs are present, they can substantially change this phenotypic association.

\section{A2. Covariance in selection response with nonzero IIGEs and genetic assortment}

We expand the covariance in evolutionary response in two species when IIGEs are present

$$
\operatorname{Cov}\left(\Delta \bar{z}_{x}, \Delta \bar{z}_{y}\right)=\mathcal{U}\left[\mathcal{A}^{2} \phi_{y x} \operatorname{Var}\left(\beta_{x x}\right)+\mathcal{B}^{2} \phi_{y x} \operatorname{Var}\left(\beta_{x y}\right)+\mathcal{C}^{2} \phi_{x y} \operatorname{Var}\left(\beta_{y y}\right)\right.
$$

$$
+\mathcal{D}^{2} \phi_{x y} \operatorname{Var}\left(\beta_{y x}\right)+\mathcal{A B} \mathcal{B} \operatorname{Cov}\left(\beta_{x x}, \beta_{x y}\right)+\mathcal{A C E} \operatorname{Cov}\left(\beta_{x x}, \beta_{y y}\right)
$$

$$
+\mathcal{A D} \mathcal{E} \operatorname{Cov}\left(\beta_{x x}, \beta_{y x}\right)+\mathcal{B} \mathcal{C} \mathcal{C} \operatorname{Cov}\left(\beta_{x y}, \beta_{y y}\right)+\mathcal{B D} \mathcal{E} \operatorname{Cov}\left(\beta_{x y}, \beta_{y x}\right)
$$

$$
\left.+\mathcal{C D E} \operatorname{Cov}\left(\beta_{y y}, \beta_{y x}\right)\right]
$$


bioRxiv preprint doi: https://doi.org/10.1101/2021.04.14.439826; this version posted September 8, 2021. The copyright holder for this preprint (which was not certified by peer review) is the author/funder, who has granted bioRxiv a license to display the preprint in perpetuity. It is made available under aCC-BY 4.0 International license.

874 where

875

876

$$
\mathcal{U}=\left(1-\phi_{x y} \phi_{y x}\right)^{-4}
$$

877

$$
\mathcal{A}=G_{x x}+G_{x y} \phi_{x y}
$$

878

$$
\mathcal{B}=G_{x y}+G_{x x} \phi_{y x}
$$

879

$$
\mathcal{C}=G_{y y}+G_{x y} \phi_{y x}
$$

880

$$
\mathcal{D}=G_{x y}+G_{y y} \phi_{x y}
$$

881

$$
\mathcal{E}=1+\phi_{x y} \phi_{x y}
$$

882 